\title{
The Surgeon and Molecular Knowledge
}

\author{
Ravi Kant • Bina Ravi
}

Published online: 6 June 2013

(C) Association of Surgeons of India 2013

The molecules in the DNA are signaling pathway and can inform us today as to what will happen to the cell in the future. The molecular signature can forecast whether it will (a) become malignant, (b) respond to certain medicines or not, (c) metastasize to lymph nodes, and (d) respond to radiation or chemotherapy or newer drugs. We can tailor our treatment in most cases using the detailed molecular knowledge. Some tumors look alike in histological slides; yet by molecular stain, we can identify each tumor in correct manner, allowing us to choose the proper treatment. Examples are confusion between (a) undifferentiated adenocarcinoma and lymphoma, (b) gastrointestinal tumor and leiomyoma, and (c) colorectal cancer metastasizing more and also resistant to some newer treatments, and (d) soft tissue sarcoma subtypes, as each subtype has a different prescription-some need more of surgery, whereas some need more of multimodality treatment. Spread of tumor is also linked to molecular and genetic knowledge as size of tumor or histology alone cannot predict the course of disease, especially in breast cancer and lymphoma. Aggressive type of tumor will need a more aggressive treatment, whereas indolent type of tumor will need a more conservative approach.

The current molecular knowledge has altered the management pathways of cancers of the breast, lung, thyroid, head and neck, gastrointestinal stromal tumors (GIST), stomach, pancreas, colon, melanoma, and soft tissue. It is essential for the surgeon to be familiar with new advances in molecular and genetic knowledge.

Breast Cancer Molecular classification of breast cancer is essential to predict (1) prognosis and (2) treatment response. Among the subtypes of breast cancer, (a) luminal A has best

R. Kant

All India Institute of Medical Sciences, Bhopal, MP, India

B. Ravi $(\bowtie)$

Lady Hardinge Medical College, University of Delhi,

New Delhi, India

e-mail: ravibina@gmail.com overall survival and best disease-free survival, apart from estrogen positive receptor status $(\mathrm{ER}+)$; (b) luminal $\mathrm{B}$ is $\mathrm{ER}+$ and Her2+ and is helped by the administration of Her2 blocker drug trastuzumab (Herceptin); (c) intermediate type is Her2 positive but ER negative and progesterone receptor (PR) negative; and (d) basal type is triple negative (ER negative, PR negative, and Her2 negative) and carries the worst prognosis. PARP inhibitors in the basal type of breast cancer are promising as conventional treatment is invariably unsuccessful. The size of a tumor is an insufficient indicator of prognosis, instead molecular classification stratifies the breast cancer in low or high risk category, avoiding overtreatment in low risk category. Gene-based assay predicts better than Adjuvant! Online, a softwarebased analytical tool.

Prognostic and predictive markers in breast cancer include IHC4. It provides a risk of recurrence based on ER, PR, Her2, and Ki67 proteins. MammaPrint is a microarraybased analysis of 70 genes in breast cancer tissue and can identify $40 \%$ of patients with low risk in comparison to the $15 \%$ that are identified with conventional methods, thus preventing numerus unnecessary under- or overtreatments. MammaPrint had the highest accuracy in predicting distant metastasis and overall survival compared to historical factors such as age, tumor size, tumor grade, and estrogen status, as well as the Adjuvant! Online software model and Nottingham and St. Gallen staging criteria. It identifies early metastasis risk with highest accuracy (RASTER study). Oncotype Dx is 21 gene analysis of breast cancer tissue (RT-PCR assay). It can predict an ER-positive, axillary node-negative patient on tamoxifen, who can benefit from chemotherapy. It strongly predicts risk of recurrence. Mammostrat Markers Mammostrat detects the presence of five proteins (SLC7A5, p53, NDRG1, TRMT2A (HTF9C), and CEACAM5). Mammostrat risk index denotes a relatively high, moderate, or low risk of recurrence. Other newer prognostic indicators include: (a) wound response gene, activation of fibroblasts and quick wound healing predict risk of metastases and death, and (b) two gene recurrence score-homeobox 13 
and IL 17B gene. High ratio between these two genes shows poor outcome. Thus, in such a scenario, tamoxifen alone will not help in T1, ER-positive, node-negative tumors, and addition of further treatment like chemotherapy will also be required.

We can predict response to therapy in early breast cancer based on tumor and host characteristics. Tumor characteristics are assessed by gene assay like Oncotype Dx (TAILORx) or by MammaPrint (MINDACT trial). Host characteristics are enzymes which are needed for metabolization of drugs. Such enzymes include CYP450-encoded enzymes-CYP 2, CYP 3, CYP2D6, and CYP2C19. A diagnostic tool like AmpliChip CYP450 by Roche can identify the presence or absence of these enzymes [1-3].

Thyroid Cancer BRAF is the new adverse prognostic marker in papillary cancer of thyroid mandating total thyroidectomy. So, even a smaller tumor with low risk profile in papillary thyroid carcinoma with BRAF will maximize treatment. PTEN and PPARy can also predict the survival of papillary thyroid cancer. RAS and CTNNB can also predict survival of follicular thyroid cancer. This information is a tool for future research on possible role of new drugs for these solid tumors; as by blocking these pathways, we can alter the course of disease $[4,5]$.

Gene-based diagnosis as well as surgery in medullary carcinoma thyroid (MCT) is the new norm. In the presence of expression of RET gene, even a 2-year-old child with normal serum calcitonin and without any visible thyroid nodule will need maximal surgery in medullary carcinoma of thyroid (MEN type 2b). The advanced MCT will need PET or somatostatin receptor scintigraphy for the detection of location. We can ablate advanced tumor by MIBG.

Head and Neck Cancer Human papilloma virus (HPV 16) results in early DNA changes in tonsillar and oral cancer. Epstein-Barr virus-associated DNA changes are seen in early stage of oral and Burkitt's lymphoma. The knowledge has resulted in new technology for early diagnosis and possible use of vaccines for prevention. The DNA-based saliva test for oral cancer is the new way of diagnosing early cancer. Premalignant phase can be diagnosed by chromosomal and molecular study. The role of HPV vaccine in selected population to prevent oral cancers is an option.

Stomach Cancer Helicobacter pylori Cag A gene expression is upregulated in the presence of intraluminal salt. H. pylori eradication can prevent secondary cancer. The genetic marker may provide a clue of early gastric cancer, much earlier than provided by conventional diagnosis methods. There is also role of antibody G17DT for prevention as well as in early reversal of gastric cancer. $\mathrm{CDH} 1$ gene is associated with familial gastric cancer. Wnt signaling pathway and E-cadherin have a major role in the development of gastric cancer [6].

Pancreas Cancer Dismal 5-year survival and poor prognosis of pancreas cancer despite heroic surgery mandate new areas of molecular research in solid tumors. New markers for diagnosis are still at experimental stage. The possible new markers include mesothelin, macrophage inhibitory cytokine-1, mitochondrial mutations, aberrant miRNA, APC and p16 gene, DNA methylation, and preneoplastic pancreatic intraepithelial neoplasia. Possible future treatment of pancreatic cancer include biologic therapy, e.g., anti-angiogenic drugs (thalidomide as anti-angiogenic agent, anti-VEGF like bevacizumab); the MMP, COX 2 and lipooxygenase inhibitors, tyrosine kinase inhibition by panitumumab, EGFR blockers, and use of drugs which alter intracellular signaling pathway (MAPK/MEK).

GIST Gastrointestinal tumor tissue diagnosis by CD117 stain has significantly diminished the true incidence of leiomyomas. PET scan is the investigation of choice in diagnosis in GIST. The tyrosine kinase pathway and receptors like platelet-derived grown factor receptor in Cajal cells mandate the role of imatinib and sunitinib in adverse prognosis or in incompletely resected GIST. The new concept of immunomodulator has changed the earlier dismal scenario. Lymph node dissection in GIST is not required unlike carcinoma of the stomach.

Colorectal Cancer DNA examination of stool is a better tool than occult blood testing for the diagnosis of early colorectal cancer, though cost and availability are the major issues. With K-ras, p53, APC, microsatellite instability (MIS), and Bat 26, the sensitivity is $91 \%$ and specificity is $93 \%$. If we exclude K-ras, then specificity is $100 \%$ in the detection of adenoma of $>1 \mathrm{~cm}$ size (sensitivity $73 \%$ and specificity $100 \%$ ). An easy explanation of the etiology of colorectal cancer classifies genes as gatekeepers, caretakers, and landscapers. On a molecular level, chromosomal instability ( $\beta$-catenin pathway, deleted in cancer gene (DCC), silent gatekeeper theory), MIS, and transforming growth factor- $\beta$ pathway are involved in colorectal cancer. The primary defect is chromosomal instability in familial adenomatous polyposis with methylation defect and mismatch repair (MMR) gene in cases of hereditary non-polyposis colon carcinoma or Lynch's syndrome. Each of six types of MMR gene like hMSH2, hMSH6, hMLH1, hMLH3, hPMS1, and hPMS2 has a different clinical spectrum and an entirely different prognosis. Molecular knowledge is essential for proper treatment and correct prognosis of colorectal cancer. New entities like DCC and familial 
cancer $\mathrm{X}$ are equally relevant to understand varied behavior of colorectal cancer.

Prognosis in colorectal cancer also depends on K-ras mutation as it denotes increased nodal metastases, higher recurrence rate, and poor survival. The $\mathrm{K}$-ras mutation (excluding wild type of K-ras) also precludes the use of cetuximab in advanced colorectal cancer. Predicting response to $5 \mathrm{FU}$ in colorectal cancer is an option if levels of thymidine synthase and thymidine phosphorylase are analyzed. Bevacizumab - an angiogenesis blocker monoclonal antibody - is also approved for addition to FOLFOX (5FU + oxaliplatin) as first line in metastatic cancer. Molecular imaging of advanced colorectal cancer by PET scan of hepatic metastases and local recurrence is beneficial. The molecular imaging, molecular diagnosis, and molecular therapy have changed the previously inoperable colorectal cancer into a possible second-look surgery and improved overall survival [7].

Soft Tissue Sarcomas The new classification and diagnostic tool is chromosomal and gene based. The chromosomal knowledge in soft tissue sarcoma is essential for optimum plan of treatment and prognosis. Mistakes in diagnosis are quite common without use of the chromosomal study [8, 9].

\section{Conclusions}

In summary, knowledge of molecular abnormalities can help us in prevention, vaccines, diagnosis, treatment, and prediction of prognosis. The knowledge of molecular pathways and the knife of the surgeon are entwined.

\section{References}

1. Sotiriou C, Pusztai L (2009) Gene-expression signatures in breast cancer. N Engl J Med 360:790-800

2. Trapé AP, Gonzalez-Angulo AM (2012) Breast cancer and metastasis: on the way toward individualized therapy. Cancer Genomics Proteomics 9(5):297-310

3. Paik S (2011) Is gene array testing to be considered routine now? Breast 20(Suppl 3):S87-S91. doi:10.1016/S0960-9776(11)70301-0

4. Wreesmann VB, Singh B (2008) Clinical impact of molecular analysis on thyroid cancer management. Surg Oncol Clin N Am 17(1):1-35

5. Kimura ET, Nikiforova MN, Zhu Z, Knauf JA, Nikiforov YE, Fagin JA (2003) High prevalence of BRAF mutations in thyroid cancer: genetic evidence for constitutive activation of the RET/PTC-RASBRAF signaling pathway in papillary thyroid carcinoma. Cancer Res 63:1454-1457

6. Blair VR (2012) Familial gastric cancer: genetics, diagnosis and management. Surg Oncol Clin N Am 21(1):35-56

7. Akiyoshi T, Kobunai T, Watanabe T (2012) Predicting the response to preoperative radiation or chemoradiation by a microarray analysis of the gene expression profiles in rectal cancer. Surg Today 42(8):713-719. doi:10.1007/s00595-012-0223-8, Epub 2012 Jun 16

8. Mertens F, Panagopoulos I, Mandahl N (2010) Genomic characteristics of soft tissue sarcomas. Virchows Arch 456(2):129-139. doi:10.1007/ s00428-009-0736-8, Epub 2009 Feb 3

9. Szuhai K, Cleton-Jansen AM, Hogendoorn PC, Bovée JV (2012) Molecular pathology and its diagnostic use in bone tumors. Cancer Genet 205(5):193-204 